\title{
Theoretical evolutionary framework for low-mass stars in massive star clusters
}

\author{
Santi Cassisi \\ INAF - Astronomical Observatory of Teramo, \\ Via M. Maggini, Teramo, 64100, Italy \\ email: cassisi@oa-teramo.inaf.it
}

\begin{abstract}
Recent spectroscopic and photometric surveys of Galactic Globular Clusters have shown that these stellar systems host distinct sub-populations of stars characterised by peculiar chemical patterns. In the following we wish to address the issue of how these specific chemical patterns affect both the structural and evolutionary properties of stars as well as their spectral energy distribution. The implications of these effects on the photometric appearance of multiple stellar populations in different photometric planes are also briefly discussed.
\end{abstract}

Keywords. stars: Population II, stars: low-mass, stars: interiors, stars: evolution

\section{Introduction}

Extensive spectroscopical surveys of Galactic GCs have shown the existence of welldefined and peculiar chemical patterns among the stars belonging to the same cluster such as the existence of light-elements (anti-) correlations - the most famous being the Na-O anti-correlation - as discussed in the reviews by Gratton et al.(2004) and Gratton, Carretta \& Bragaglia(2012). In the same time, very accurate photometric investigations performed by means of the HST have revealed the existence in the Color-Magnitude Diagram (CMD) of various GCs of multiple sequences such as distinct Main Sequence (MS) and Sub-Giant Branch (SGB) loci, and multiple Red Giant Branch (RGB) loci. Actually, the features observed in the CMD change significantly from a cluster to another one, and their properties strongly depend on the adopted photometric systems (see Piotto 2010 and Piotto et al. 2015 and references therein).

The commonly accepted scenario is that, in any GC, a second (and in some cases also more) generation(s) of stars can form from the ejecta of intermediate-mass and/or massive stars belonging to the first stellar population formed during the early phase of the cluster evolution, whose initial chemical composition is modified by high-temperature proton captures. Although some amount of dilution between pristine (unpolluted) matter and nuclearly processed matter seems to be unavoidable in order to explain many observational evidence, the second generation stars would be formed via matter characterised by light-element (anti-)correlations and He enhancement.

The ability to trace both spectroscopically and photometrically the various sub-populations hosted by each individual GC, allows now the identification of both the primordial stellar component (the first generation, FG) and the second generation (SG) stars. The existence of a strong correlation between the spectroscopic signatures of the distinct subpopulations and their distribution along the multiple CMD sequences, has suggested that the peculiar chemical patterns of SG stars have to affect both the evolutionary properties of these stars as well as their spectral energy distribution (SED).

The investigations performed so far in order to understand the physical reasons for the occurrence of these multiple evolutionary sequences in the CMD of GCs have provided 
sound evidence of the fact that the distinct sequences can be interpreted as due to 'quantised' He abundances such as in the case of the massive GCs $\omega$ Cen and NGC 2808, distinct CNO abundance pattens as for M 22 and of NGC1851, and the presence of light-elements anti-correlations.

It is evident that in order to properly trace the various sub-populations in a given GCs, the availability of an evolutionary theoretical framework properly - and self-consistently - accounting for the observed peculiar chemical patterns is mandatory. In this last decade several theoretical investigations have been performed in order to investigate the effects of the chemical patterns characteristic of the multiple population phenomenon on both the evolutionary and structural properties of stars (Salaris et al. 2006, Cassisi et al. 2008,Pietrinferni et al. 2009,Vandenberg et al. 2012) and model atmospheres and, hence on the corresponding color - $\mathrm{T}_{\text {eff }}$ transformations and bolometric corrections (Sbordone et al. 2011, Cassisi et al. 2013).

\section{On the impact of the peculiar chemical patterns of SG stars on stellar evolution}

He-enhancement: Helium is one of the most important chemical species in the context of stellar evolution, because any change of its abundance hugely affects the structural and evolutionary properties of stars. In more detail, a change of the He abundance affects lowtemperature, radiative opacities, for an increase of He causes a reduction of the opacity. This is due to the fact that when increasing the helium abundance at fixed metallicity, the $\mathrm{H}$ abundance has to decrease, and given that $\mathrm{H}$ is a major opacity source via the ${ }^{-} \mathrm{H}$ ion, this causes a global reduction of the opacity. This effect explains why He-enhanced stellar models have hotter $\mathrm{T}_{\text {eff }}$ values in comparison with normal He abundance stars.

The opacity reduction due to He-enhancement contributes also to make brighter the stars during the core H-burning stage, although the larger contribution to the change in the stellar surface luminosity is due to the change in the mean molecular weight associated to the $\mathrm{He}$ abundance increase. In fact, the $\mathrm{H}$-burning efficiency is strongly dependent on the value of the mean molecular weight $\mu: \mathrm{L}_{\mathrm{H}} \propto \mu^{7}$. When He increases the mean molecular weight (at fixed metallicity) has to increase and this translates in a larger H-burning efficiency. Given that $\Delta \mathrm{L}_{\mathrm{H}} / \mathrm{L}_{\mathrm{H}}=7 \Delta \mu / \mu$, a change $\Delta Y=0.10$ - lower than the maximum He enhancements expected for the bluest MS stars in GCs $\omega$ Cen (King et al. 2012) and NGC 2808 (Piotto et al. 2007, Milone et al. 2012) - causes a $\sim 50 \%$ variation of the H-burning efficiency compared to normal-He stars.

The combined effect of radiative opacity decrease and H-burning efficiency increase causes He-rich stellar models to be brighter and hotter during the MS stage. As a consequence, their core H-burning lifetime $\left(t_{H}\right)$ is significantly reduced: for a $0.8 M_{\odot}, t_{H}$ decreases by $\sim 48 \%$ when increasing the He content from the primordial value $\mathrm{Y}=0.245$ to 0.40 .

Figure 1 shows isochrones computed for the same $[\mathrm{Fe} / \mathrm{H}]$ value and age, but distinct values for the initial He content. There are some interesting features disclosed by this plot: i) the He-rich MS runs parallel in the luminosity interval from the MS Turn-off (TO) and the location of stars with mass $\sim 0.5 M_{\odot}$; ii) the MS effective temperature is sensitive to the He increase $\left(\Delta \mathrm{T}_{\text {eff }} / \Delta \mathrm{Y} \approx 2.3 \times 10^{3} \mathrm{~K}\right)$; iii) for any given age, the SGB is not affected by a He change; $v$ ) the $\mathrm{T}_{\text {eff }}$ values of RGB stellar models are also affected by an He increase, although to a smaller extent than the MS locus. The predicted behaviour of the MS as a function of the initial He abundance justifies the interpretation of the MS splitting observed in the optical CMDs of some GCs, like $\omega$ Cen and NGC 2808 (see also the following discussion on this issue). 


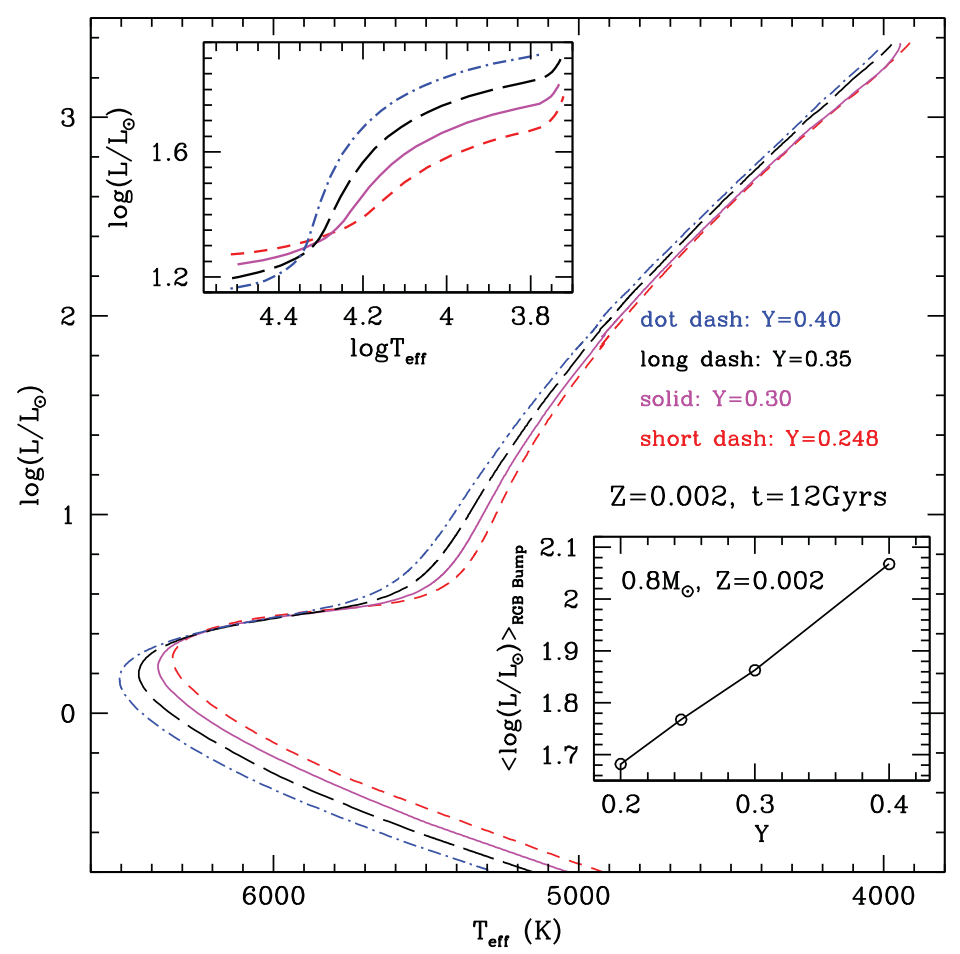

Figure 1. 12 Gyr-old, $\mathrm{Z}=0.002$, isochrones computed for various assumptions about the initial He abundance. The lower inset shows the trend of the average RGB bump brightness as a function of the initial He abundance for a $0.8 \mathrm{M}_{\odot}$ model, while the upper inset shows the location in the H-R diagram of ZAHB loci corresponding to various initial He abundances but for the same assumption about the mass of the RGB progenitor, $\mathrm{M}_{\mathrm{RGB}}=0.8 \mathrm{M}_{\odot}$, namely.

It is also worth noting that, at fixed age, the mass at the MS TO significantly decreases when increasing the initial He content: for a $12 \mathrm{Gyr}$ old isochrone it is equal to $0.806 M_{\odot}$ for $\mathrm{Y}=0.245$ and $0.610 M_{\odot}$ for $\mathrm{Y}=0.40$. As it will be discussed in the following, this occurrence has an important implication during the core He-burning stage.

When considering the RGB evolution, there are two important features that are affected by a change of the initial He abundance: the RGB bump and the brightness of the RGB tip (TRGB). Model computations predict: a) an increase of the bump brightness (see Fig. 1), and b) a smaller luminosity excursion during the RGB bump stage. The first effect is due to the lower envelope opacity of He-rich stars, that causes the discontinuity of the $\mathrm{H}$ abundance left over by the first dredge-up to be located in more external layers. As a consequence, the H-burning shell encounters the discontinuity at later times, hence at a brighter luminosity. The second effect is caused by the fact that in He-rich stars, the jump of the $\mathrm{H}$ abundance at the discontinuity is smaller than in normal $\mathrm{He}$ stars. As a consequence the surface stellar luminosity is less affected when the H-burning shell crosses the discontinuity. From an observational point of view, the impact of an He enhancement on the RGB bump brightness in GCs has been discussed by Bragaglia et al. (2010) .

The TRGB brightness is significantly affected by an He-enhancement. For a given total stellar mass increase of the initial He content decreases the TRGB brightness. This is due to the fact that the interiors of He-rich stars are hotter at the end of the core H-burning stage, and when they reach the RGB stage they develop a significantly lower level of 
electron degeneracy in their He core, as well as they are less affected by neutrino energy losses. At the same time, as a consequence of the larger H-burning efficiency, the He core mass grows at a faster rate. Both effects make 'easier' to achieve the thermal conditions required by the $3 \alpha$ reaction ignition, and He ignition is attained with a smaller He core mass (for more details see Cassisi \& Salaris 2013). Due to the existence of a He core mass - luminosity relation for RGB stars, the TRGB brightness decreases in He-rich, low-mass giants. Unfortunately, due to the low number of stars populating the brighter portion of the RGB and the still unsettled issue of the GC distance scale, it is almost impossible to verify observationally this prediction.

We also note that, as a consequence of the reduction of the value of $t_{H}$ for He-rich stars, the mass of stars at the TRGB is expected to be significantly smaller in He-enhanced stellar populations, when age is kept constant (and if RGB mass loss does not have a strong dependence on He). This occurrence explains why He-enhanced stellar populations are characterised by a, 'on average', bluer Horizontal Branch (HB) morphology with respect to a FG stellar population.

When moving to the core He-burning stage, one notices that Zero Age HB (ZAHB) brightness is a strong function of the initial He content. When Y increases, the ZAHB becomes brighter for $\mathrm{T}_{\text {eff }}$ lower that $\sim 20000 \mathrm{~K}$, and fainter at higher $\mathrm{T}_{\text {eff }}$ (see Fig. 1 ). This behaviour is the consequence of both the decrease of the He-core mass at the TRGB, and of the increased efficiency of the shell H-burning in He-rich stars. In ZAHB objects cooler than $\sim 20000 \mathrm{~K}$, the second effect dominates over the first one and the stars appear brighter, whereas in the hottest portion of the ZAHB - due to the tiny envelope mass the H-burning shell is not efficient enough, and the decrease of the He core mass is the dominating effect. This has the important implication that the slope of the ZAHB in the $\mathrm{H}-\mathrm{R}$ diagram (as well as in the various observational planes) is strongly dependent on the (spread in the) initial He abundances of the various sub-populations hosted within a given GC. This theoretical prediction can provide a direct explanation for the existence of a tilted HB in GCs like NGC6388, NGC6441 and NGC1851.

He-enhancement has an additional important implication for the global HB morphology. Due to the combined effect of the lower RGB evolving mass and more extended blue loops that characterise the off-ZAHB evolution of He-rich stars, for a given average efficiency of the mass loss along the RGB the predicted HB location of He-enhanced models will be on average hotter, i.e. bluer, than that of He-normal stars. This helps explaining the existence the very blue HB morphology - as well as the presence of extended HB blue tail - in GCs hosting He-enhanced sub-populations. Therefore, it appears evident that the presence of He-enhanced stellar populations in Galactic GCs can contribute to solve the longstanding 'second parameter problem', i.e. the presence of different HB morphology among clusters with the same metallicity. This issue has been recently discussed by Milone et al.(2014).

Light-element (anti-) correlations: light element abundance changes can affect the stellar properties via the effects induced on both the radiative opacity evaluations and - at least in the case of $\mathrm{C}, \mathrm{N}$ and $\mathrm{O}$, i.e. the CNO-cycle catalysts - the H-burning efficiency. Many investigations have been devoted to this topic as those by Salaris et al.(2006), Cassisi et al.(2008), Pietrinferni et al.(2009), Ventura et al.(2009), Vandenberg et al.(2012) and the main results can be summarised as follows:

- even in case of extreme light-element anti-correlations, as long as the sum of CNO elements is kept constant, the evolution in the H-R diagram is unchanged compared to standard $\alpha$-enhanced stellar models;

- if the CNO sum is enhanced, the morphology of the evolutionary tracks in the H-R diagram is modified. For a given iron content and stellar mass, the SGB appears fainter 

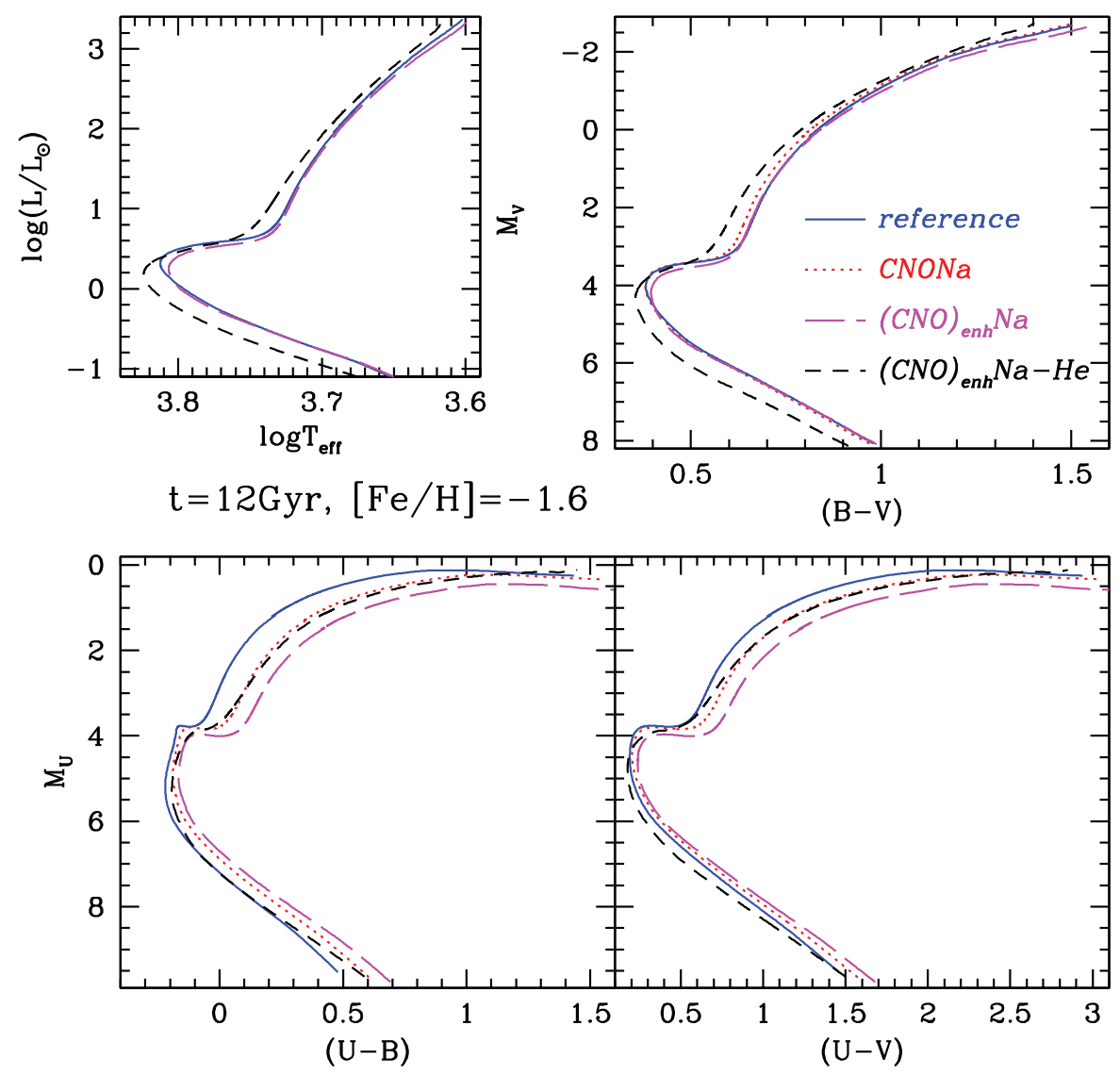

Figure 2. Theoretical isochrones for the same age and metallicity (see label) but various assumptions about the light-element distribution and He-enhancement: reference: canonical $\alpha$-enhanced mixture, $\mathrm{Y}=0.248 ; C N O N a$ : light-element (anti-)correlation but the same CNO sum and $\mathrm{He}$ abundance of reference mixture; $(C N O)_{e n h} N a$ : as before but the CNO sum is enhanced by a factor $\sim 2 ;(C N O)_{e n h} N a-H e$ : as before but now the initial He abundance is equal to $\mathrm{Y}=0.40$.

in comparison with standard $\alpha$-enhanced models. This behaviour is mainly due to the fact that the efficiency of the CNO-cycle increases when the CNO sum is enhanced. The $\mathrm{T}_{\text {eff }}$ scale for both MS and RGB CNO-enhanced stellar models is marginally affected (by less than $20 \mathrm{~K}$ );

- when computing isochrones, unless the CNO sum is increased with respect the reference $\alpha$-enhanced mixture, the effect of the light-element (anti-)correlations on the H-R diagram location is negligible. When the $\mathrm{C}+\mathrm{N}+\mathrm{O}$ sum is increased, a separation appears along the SGB. When enhancing by a factor of $\sim 2$ the CNO sum, a CNO-enhanced isochrone is almost perfectly mimicked by a 'canonical' $\alpha$-enhanced, $\sim(1.5-2)$ Gyr older, isochrone.

\section{On the impact of SG chemical patterns on the SED}

As shown in the upper, left panel of fig. 2, in the theoretical H-R plane, the only possibility to observe a separation between isochrones with a standard $\alpha$-enhanced isochrone and those with the chemical composition of SG stars, is to assume a huge He 
enhancement (that affect MS and RGB) and/or a significant CNO-enhancement (that affect the SGB sequence). Needless to say that this theoretical evidence is in contrast with observations, that reveal the presence of splitting/broadening of the photometric sequences (in particular, the RGB one) also in those GCs that do not show (spectroscopical) evidence of a significant CNO enhancement and/or huge He enhancement. The chemical peculiarities of second generation stars must therefore have an effect also on the spectral energy distribution.

A detailed analysis of the impact of the peculiar chemical patterns of SG stars on their SED has been performed by Sbordone et al.(2011), Cassisi et al.(2013), and Dotter et al.(2015). Figure 3 summarises the results of their investigation displaying the comparison between synthetic spectra for a specific SG composition computed for selected MS, TO and RGB models covering the wavelength range from 200 to $1000 \mathrm{~nm}$, and theoretical spectra for the same stars but with a FG chemical composition. Some prominent absorption features (mostly molecular bands), whose strength changes significantly between FG and SG compositions are labelled, and the transmission curves for the Johnson-Cousins UBVI, and the Strömgren uvby filters are also displayed.

Starting with the RGB fluxes, the SG mixture shows much stronger NH and CN absorption bands in the U, B, I passbands compared to the FG one. This is due to the higher $\mathrm{N}$ abundance, despite the fact that the $\mathrm{C}$ abundance is lower, because the $\mathrm{N}$ abundance acts as bottleneck in forming $\mathrm{CN}$ molecules. The G-band - a CH feature within the $\mathrm{B}$ passband - is stronger in the FG mixture, because of the higher $\mathrm{C}$ abundance. The increased opacity in the blue part of the SG spectrum increases the continuum flux redwards of $\sim 450 \mathrm{~nm}$.

When moving to the spectrum of a MS TO star, the much higher temperature prevents the formation of $\mathrm{CN}$ and $\mathrm{CH}$ molecules, as well as most of the effect on the G-band. Only the very strong $\mathrm{NH}$ band around $340 \mathrm{~nm}$ is still visible. The cool MS star displays again features similar to the RGB one, with a much stronger $\mathrm{OH}$ absorption at the blue edge of the U filter in the FG flux distribution. The NaD appears much stronger in the SG spectrum, for the $\mathrm{Na}$ abundance is increased due to the O-Na anti-correlation.

On the other hand, it has been found that the impact of $\mathrm{Mg}-\mathrm{Al}$ abundance variations at fixed $[\mathrm{Fe} / \mathrm{H}]$ and helium abundance is negligible on stellar models and isochrones (from the main sequence to the tip of the red giant branch) and bolometric corrections, when compared to the effect of $\mathrm{C}-\mathrm{N}$ and $\mathrm{O}-\mathrm{Na}$ variations.

These results show the crucial role played by $\mathrm{CN}$ and $\mathrm{NH}$ molecules (whose abundances in SG stars are very different from those in FG stars) in modifying the stellar spectrum at wavelengths shorter than $\sim 400 \mathrm{~nm}$. This has the important implication that only magnitudes corresponding to photometric filters bluer than the standard Johnson B filter are affected by the peculiar chemical patterns of multiple populations; as a consequence, the photometric appearance of these sub-populations depends on the adopted photometric systems. How theoretical isochrones suitable for SG chemical compositions compare with isochrones appropriate for standard $-(\mathrm{FG})$ chemical abundances in the CMD in various photometric systems can be summarised as follows:

- Visible to near-infrared CMDs (e.g. BVI or Strömgren vy) are generally unaffected by the presence of SG stars, unless this stellar component has a different initial He content (see the upper, right panel in fig. 2). In this case multiple sequences appear along the MS. This behaviour explains very nicely the multiple MS in the optical CMD of NGC 2808 discussed by Piotto et al.(2007). A variation of the CNO sum also affects optical CMDs by altering the location of TO and SGB. This occurrence provides a sound interpretation for the double SGB of NGC 1851 in optical filters (see Cassisi et al. 2008), as well as for the double SGB observed in NGC 6656 (Marino et al. 2012). Both He and CNO affect 

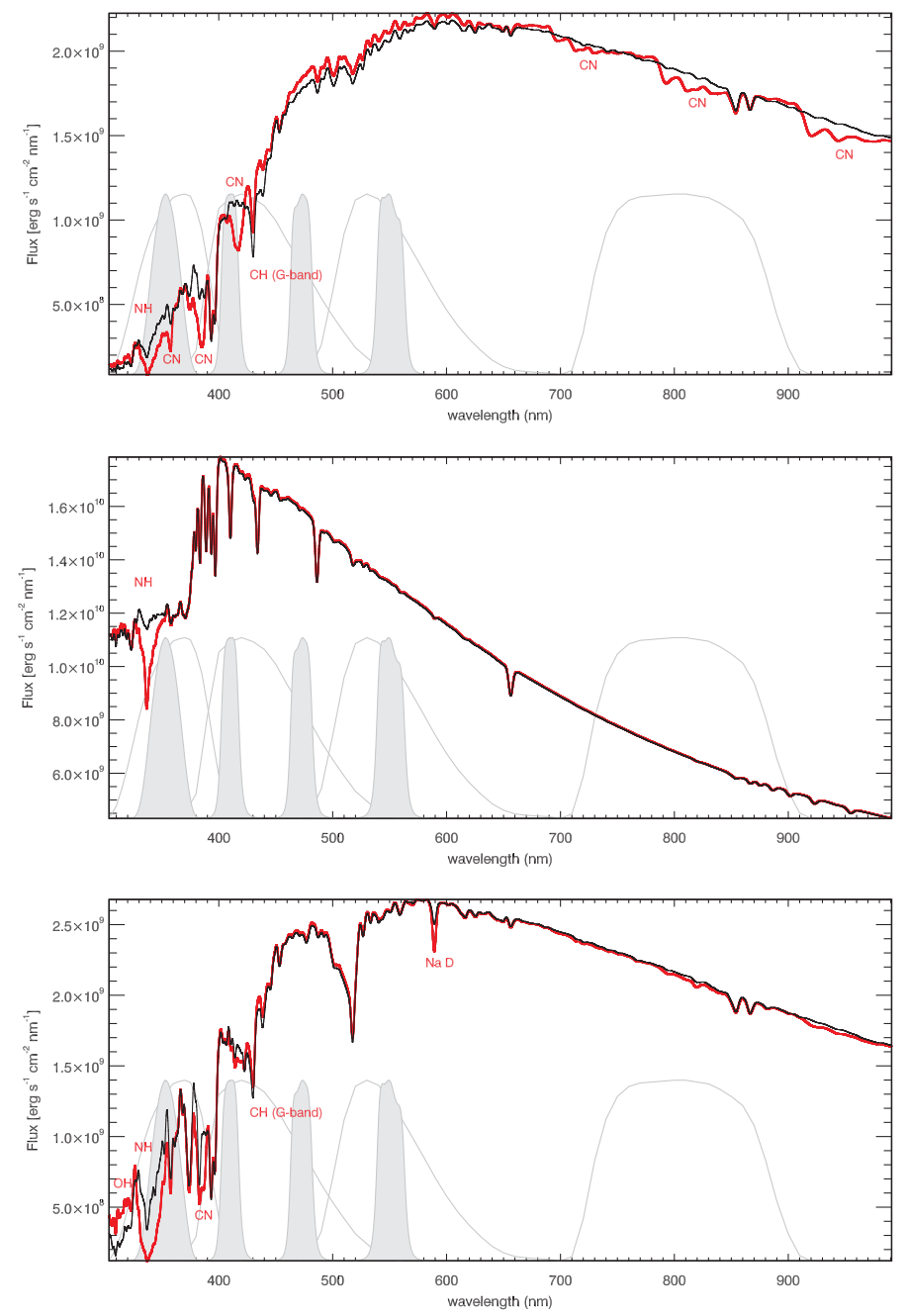

Figure 3. Synthetic spectra for a reference mixture (thin black line) and $(C N O)_{e n h} N a$ mixture (red thick line) for selected stellar models. The top panel displays the spectra for a RGB model with $\mathrm{T}_{\text {eff }}=4476 \mathrm{~K}, \log (\mathrm{g})=4.1 .2$, the middle panel panel the flux for a MS TO model with $\mathrm{T}_{\text {eff }}=6490 \mathrm{~K}, \log (\mathrm{g})=4.22$, while the bottom panel corresponds to a MS model with $\mathrm{T}_{\text {eff }}=4621 \mathrm{~K}, \log (\mathrm{g})=4.47$. The transmission curves for the Johnson-Cousins UBVI filters (thin, black lines) and for the Strömgren uvby filters (grey-shaded regions) are also displayed (adapted from Cassisi \& Salaris 2013).

optical CMDs because they hugely affect the stellar evolutionary properties, and hence modify the isochrone location in the H-R diagram.

- Optical-UV CMDs (e.g. UBV, UVIS@WFC3, and Strömgren uy) are hugely affected by the presence of SG stars. In fact, $\mathrm{C}-\mathrm{N}-\mathrm{O}-\mathrm{Na}$ anti-correlations affect the bolometric correction scale for photometric passbands bluer than $\sim 400 \mathrm{~nm}$, so that even at constant CNO sum and He abundance, SG and FG stars will be distributed amongst several separate sequences. The quantitative effect will depend on the filter combination employed and the exact over- and under-abundances with respect to the baseline FG composition. Variations of He and CNO sum add an additional level of separation in the CMDs, as a 
consequence of their effect on the underlying isochrones as shown in the bottom panels of fig. 2 .

\section{Acknowledgements}

SC warmly thanks the organizers for inviting him to this interesting and pleasant conference. Financial support from PRIN INAF 2014 (PI: S. Cassisi) and PRIN MIUR 2010-2011, project "The Chemical and Dynamical Evolution of the Milky Way and Local Group Galaxies", prot. 2010LY5N2T" is acknowledged.

\section{References}

Bragaglia, A., Carretta, E., Gratton, R., D’Orazi, V., Cassisi, S., \& Lucatello, S. 2010, A\&A, 519,60

Cassisi, S., Salaris, M., Pietrinferni, A., Piotto, G., Milone, A. P., Bedin, L. R., \& Anderson, J. 2008, ApJ (Letters), 672, L115

Cassisi, S., Mucciarelli, A., Pietrinferni, A., Salaris, M., \& Ferguson, J. 2013, A\&̧A, 554, A19

Cassisi, S. \& Salaris, M. 2013, Old stellar populations: how to study the fossil record of galaxy formation, Wiley- $\mathrm{VCH}$

Dotter, A., Ferguson, J. W., Conroy, C., et al. 2015, MNRAS, 446, 1641

Gratton, R., Sneden, C., \& Carretta, E. 2004, ARAA, 42, 385

Gratton, R., Carretta, E., \& Bragaglia, A. 2012, A\&ARv, 20, 50

King, I. R., Bedin, L. R., Cassisi, S., et al. 2012, AJ, 144, 5

Marino, A. F., Milone, A. P., Sneden, C., et al. 2012, A\&A, 541, A15

Milone, A. P., Piotto, G., Bedin, L. R., et al. 2012, A\&A, 537, 77

Milone, A. P., Marino, A. F., Dotter, A., et al. 2014, ApJ, 785, 21

Pietrinferni, A., Cassisi, S., Salaris, M., Percival, S., \& Ferguson, J. W. 2009, ApJ, 697, 275

Piotto, G. Bedin, L. R., Anderson, J., et al. 2007, ApJ (Letters), 661, L53

Piotto, G. 2010, Publications of the Korean Astronomical Society, 25, 91

Piotto, G., Milone, A. P., Bedin, L. R. et al. 2015, AJ, 149, 91

Salaris, M., Achim, W., Ferguson, J. W., \& Fusilier, D. J. 2006, ApJ, 645, 1131

Sbordone, L., Salaris, M., Weiss, A., \& Cassisi, S. 2011, A\&A, 534, 9

VandenBerg, D. A., Bergbusch, P. A., Dotter, A., Ferguson, J. W., Michaud, G., Richer, J., \& Proffitt, C. R. 2012, ApJ, 755, 15

Ventura, P., Caloi, V., D’Antona, F., Ferguson, J., Milone, A. P., \& Piotto, G. P. 2009, MNRAS, 399, 934 